\title{
Correlation of hypertensive disorders in pregnancy with procedures of in vitro fertilization and pregnancy outcomes
}

\author{
FANG XIONG, LINGQING HU, YUN ZHANG and XIAO XIAO \\ Center of Reproduction Medicine, Maternal and Child Health Hospital of Wuxi, Wuxi, Jiangsu 214002, P.R. China
}

Received May 18, 2017; Accepted September 19, 2017

DOI: 10.3892/etm.2017.5204

\begin{abstract}
We investigated the correlation of hypertensive disorders in pregnancy with different procedures of in vitro fertilization, and analyzed pregnancy outcomes of patients with hypertensive disorders in pregnancy. A retrospective analysis was performed on the medical records of 658 maternity patients who conceived through in vitro fertilization in Maternal and Child Health Hospital of Wuxi. Patients were divided into two groups according to different fertilization procedures: i) the routine in vitro fertilization-embryo transfer group (IVF-ET group, 377 cases) and ii) intra-cytoplasmic sperm injection-embryo transfer group (ICSI-ET group, 281 cases). Consequently, patients were further divided into two groups according to different embryo transfer cycles: the fresh embryo transfer group (F-ET group, 446 cases) and the frozen-thawed embryo transfer group (T-ET group, 212 cases). Characteristics of patients in each group were evaluated, and incidence of hypertensive disorders in pregnancy resulting from different assisted reproductive technology was compared. Among patients who conceived through IVF, there were 56 cases of hypertensive disorders in pregnancy, including 21 cases of gestational hypertension, 34 cases of pre-eclampsia and 1 case of eclampsia. The odds ratio (OR) of gestational hypertension in the comparison between the ICSI-ET and IVF-ET groups was 2.01 (0.81-4.74), and was reduced to 1.69 (0.70-4.02) after correction. The difference of OR in twinbirth patients of the two groups was statistically significant, but the difference in single-birth patients was not statistically significant. The odds ratio (OR) of gestational hypertension in the comparison between the F-ET and T-ET groups was 0.44 (0.13-1.34), and became 0.49 (0.15-1.51) after correction. The odds ratio of pre-eclampsia in the comparison between the ICSI-ET and IVF-ET groups was $1.36(0.42-4.18)$, and was reduced to $1.17(0.36-3.62)$ after correction. The odds ratio of
\end{abstract}

Correspondence to: Dr Fang Xiong, Center of Reproduction Medicine, Maternal and Child Health Hospital of Wuxi, 48 Huaishu Street, Wuxi, Jiangsu 214002, P.R. China

E-mail: xiong_fang1@163.com

Key words: test-tube baby, gestational hypertension, pre-eclampsia, embryo transfer cycle pre-eclampsia in the comparison between the F-ET and T-ET groups was 0.93 (0.42-1.96), and became 0.98 (0.44-2.12) after correction. The differences were not statistically significant. The risk of onset of hypertensive disorders in pregnancy has a certain correlation with the ICSI fertilization technology, but has no apparent correlation with embryo transfer cycles.

\section{Introduction}

Presently, approximately $10 \%$ of couples are plagued by reproduction-related issues mainly caused by environmental factors and stress. Since the advent of assisted reproductive technology such as the in vitro fertilization-embryo transfer, artificial insemination, and ovulation induction techniques, provided great opportunity for infertile patients to coup with their problem.

However, it has been reported that IVF technology may have certain undesirable side effects, such as OHSS and hypertensive disorders in pregnancy, possibly due to use of drugs and operational intervention $(1,2)$. Hypertensive disorders in pregnancy have an insidious onset with a fast progressive course, which may cause serious challenges (3), and may threaten maternal and neonatal health. Studying the connection between IVF procedures and hypertensive disorders in pregnancy can contribute to early prevention and treatment. In this study, correlation of hypertensive disorders in pregnancy with different procedures of in vitro fertilization was explored, and pregnancy outcomes of patients were evaluated.

\section{Patients and methods}

Subjects. From February 2012 to February 2016, a total of 658 maternity patients who conceived through in vitro fertilization in our hospital's assisted reproductive center and gave birth in Maternal and Child Health Hospital of Wuxi and had completed clinical records were enrolled in this study. This study was approved by the Ethics Committee of Maternal and Child Health Hospital of Wuxi. Signed written informed consents were obtained from the patients' guardians. Inclusion criteria: Patients with no history of hypertension prior to pregnancy; patient with complete clinical data in our hospital's assisted reproductive center and obstetric department; pregnancy outcomes were either live single birth or live twin births (including embryo reduction in the early stage of multiple pregnancy); patients delivered no less than 25 gestational weeks 
Table I. General data of patients in different groups $($ mean \pm SD).

\begin{tabular}{|c|c|c|c|c|c|c|c|c|}
\hline \multirow[b]{2}{*}{ Group } & \multirow[b]{2}{*}{$\mathrm{N}$} & \multirow{2}{*}{$\begin{array}{l}\text { Infertility } \\
\text { years }\end{array}$} & \multirow{2}{*}{$\begin{array}{l}\text { Gestational } \\
\text { weeks }\end{array}$} & \multicolumn{2}{|c|}{$\begin{array}{l}\text { Pregnancy } \\
\text { outcome }\end{array}$} & \multirow{2}{*}{$\begin{array}{c}\text { Birth } \\
\text { weight }(\mathrm{g})\end{array}$} & \multirow{2}{*}{$\begin{array}{c}\text { Natural } \\
\text { labor }\end{array}$} & \multirow{2}{*}{$\begin{array}{c}\mathrm{BMI} \\
\left(\mathrm{kg} / \mathrm{m}^{2}\right)\end{array}$} \\
\hline & & & & Single & Twin & & & \\
\hline IVF-ET & 377 & $4.2 \pm 3.6$ & $27.8 \pm 7.1$ & 261 & 116 & $2802.7 \pm 754.1$ & 19 & $21.8 \pm 2.7$ \\
\hline ICSI-ET & 281 & $4.4 \pm 3.3$ & $28.0 \pm 6.7$ & 198 & 83 & $2789.2 \pm 800.9$ & 16 & $21.5 \pm 3.1$ \\
\hline t-value $/ \chi^{2}$ & & 1.125 & 1.076 & \multicolumn{2}{|c|}{0.968} & 1.324 & 1.401 & 1.434 \\
\hline P-value & & 0.611 & 0.612 & \multicolumn{2}{|c|}{0.664} & 0.087 & 0.081 & 0.075 \\
\hline F-ET & 446 & $4.3 \pm 3.5$ & $27.6 \pm 6.9$ & 307 & 139 & $2792.6 \pm 746.8$ & 24 & $21.9 \pm 2.5$ \\
\hline T-ET & 212 & $4.5 \pm 3.2$ & $27.9 \pm 7.0$ & 152 & 60 & $2787.5 \pm 781.3$ & 11 & $21.6 \pm 2.9$ \\
\hline $\mathrm{t}$-value $/ \chi^{2}$ & & 1.123 & 1.254 & \multicolumn{2}{|c|}{1.106} & 1.076 & 1.365 & 1.563 \\
\hline P-value & & 0.598 & 0.058 & \multicolumn{2}{|c|}{0.421} & 0.587 & 0.088 & 0.069 \\
\hline
\end{tabular}

Table II. Single or multiple births in different groups (n, \%).

\begin{tabular}{|c|c|c|c|c|}
\hline \multirow[b]{2}{*}{ Births } & \multicolumn{2}{|c|}{ Fertilization mode } & \multicolumn{2}{|c|}{ Transfer cycles } \\
\hline & IVF-ET group & ICSI-ET group & F-ET group & T-ET group \\
\hline Single & $261(56.86)$ & $198(43.14)$ & 307 (66.88) & $152(33.12)$ \\
\hline Twin & $116(58.29)$ & 83 (41.71) & $139(69.85)$ & $60(30.15)$ \\
\hline t-value & 1.256 & 1.387 & 1.439 & 1.402 \\
\hline P-value & 0.713 & 0.621 & 0.589 & 0.615 \\
\hline
\end{tabular}

In comparison of single or twin births, $\mathrm{P}>0.05$ between IVF-ET and ICSI-ET groups and between F-ET and T-ET groups.

with a birth weight of no less than 700 grams. A specialized physician performed the whole process of ovulation induction, egg retrieval, IVF and embryo transfer. All patients had an average infertility duration of $4.3 \pm 3.5$ years, with 242 patients in primary infertility and 416 patients in secondary infertility. Patients who were diagnosed with other conditions included 39 cases of gestational diabetes, 76 cases of hepatitis B virus carriers and hepatitis B patients with symptoms, 57 cases of uterine fibroids, 9 cases with history of tuberculosis, 3 cases with history of syphilis, and 10 cases with history of thyroid disease. Patients were divided into two groups according to different fertilization procedures: i) the routine in vitro fertilization-embryo transfer group (IVF-ET group, 377 cases) and ii) the intra-cytoplasmic sperm injection-embryo transfer group (ICSI-ET group, 281 cases). Patients were also divided into two groups according to different embryo transfer cycles: the fresh embryo transfer group (F-ET group, 446 cases) and the frozenthawed embryo transfer group (T-ET group, 212 cases). Patients' general data in different groups are presented in Table I. No significant differences between the two groups $(\mathrm{P}>0.05)$ in comparison of infertility years, gestational weeks, pregnancy outcomes (single/twins), birth weight and delivery mode were detected. All corresponding data were comparable. If $\mathrm{P}<0.05$, the difference was considered statistically significant.

Methods. Diagnostic criteria for hypertensive disorders in pregnancy were based on the Gynecological Guidelines introduced by the American Association of Obstetricians and Gynecologists in 2004.

Procedures performed on patients in the IVF-ET group. Follicles were prepared using hormone replacement therapy (HRT), micro-stimulation, natural cycle and various controlled superovulation schemes. When the follicle diameter reached 18-20 mm, appropriate amount of human chorionic gonadotropin (HCG) was injected at night to trigger ovulation. Eggs were retrieved $36 \mathrm{~h}$ later through type-B ultrasound-guided needle puncture. Sperms were collected via masturbation, or surgical extraction from testicular tissue or epididymis. Best sperms and eggs were selected and put together in a culture dish allowing them combined to form fertilized eggs. After 2-5 days, the fertilized eggs in the culture dish grew to early embryos which were transferred into the patient's uterine cavity, aiming for a successful pregnancy and childbirth.

Procedures performed on patients in the ICSI-ET group. Procedures of ovulation induction, egg retrieval and sperm collection were the same as described for patients in the IVF-ET group, but the fertilization mode was different. Under a microscope, a microinjection needle was used to manually pierce through the zona pellucida and egg cell membrane to inject a single sperm into the cytoplasm for fertilization. Compared with routine IVF, ICSI had a higher success rate of pregnancy. ICSI was beneficial to patients suffering from 
Table III. Child-bearing age of patients in different groups (n, \%).

\begin{tabular}{|c|c|c|c|c|c|c|}
\hline Age range & IVF-ET group & ICSI-ET group & F-ET group & T-ET group & $\chi^{2}$-value & P-value \\
\hline $20-24$ & $6(1.60)$ & $4(1.42)$ & $8(1.79)$ & $3(1.42)$ & 1.865 & 0.726 \\
\hline $25-29$ & $89(23.60)$ & $74(26.33)$ & $103(23.09)$ & $46(21.70)$ & 2.013 & 0.613 \\
\hline $30-34$ & $166(44.03)$ & $130(46.26)$ & $201(45.07)$ & $96(45.28)$ & 1.432 & 0.941 \\
\hline $35-39$ & $84(22.28)$ & $57(20.28)$ & $100(22.42)$ & $47(22.17)$ & 1.648 & 0.827 \\
\hline $40-45$ & $32(8.49)$ & $16(5.69)$ & $34(7.62)$ & $20(9.43)$ & 2.369 & 0.525 \\
\hline
\end{tabular}

There were no significant differences in each age range between groups $(\mathrm{P}>0.05)$.

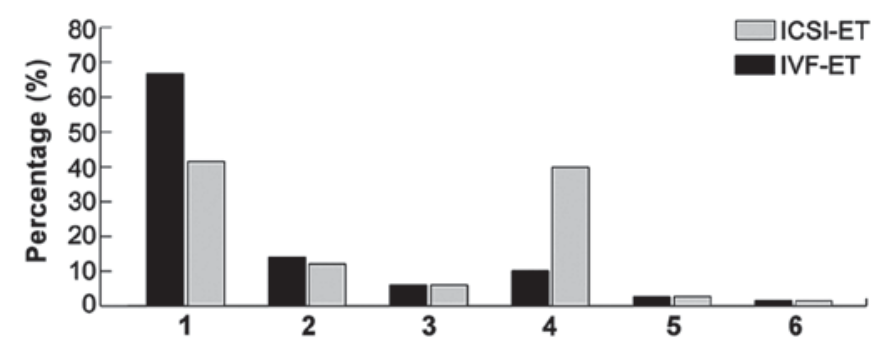

Figure 1. Causes of infertility and fertilization modes (1, tubal problems; 2, endometriosis; 3 , ovulation disorders; 4, male sperm abnormalities; 5 , other factors; 6 , unknown causes).

infertility due to serious male oligoasthenoteratozoospermia, obstructive azoospermia, and nonfertilization after routine IVF.

Procedures performed on patients in the F-ET group. Fresh embryos were transferred into the patient's uterine cavity after 2-5 days following fertilization under above-mentioned fertilization modes.

Procedures performed on patients in the T-ET group. In some patients, fresh embryo transfer was given up due to OHSS, peak level of progesterone or other reasons. After this cycle, frozen-thawed embryo transfer was performed.

Statistical method. Statistical analysis was performed using SPSS 18.0 software (SPSS Inc., Chicago, IL, USA). Measurement data were expressed as mean $\pm \mathrm{SD}$, and compared using $t$ inspection. Ratios and rates of two groups and above were compared using the exact probability test of a contingency table. The binary regression model was used to calculate uncorrected odds ratio (OR) and confidence interval (CI), as well as corrected OR and CI by multiple factors. Interference of confounding factors was avoided as far as possible. Difference was considered statistically significant when $\mathrm{P}<0.05$.

\section{Results}

Characteristics of patients who conceived through assisted reproductive technology

Single or multiple births in different groups. For single birth, 261 cases $(56.86 \%)$ and 198 cases $(43.14 \%)$ were recorded in

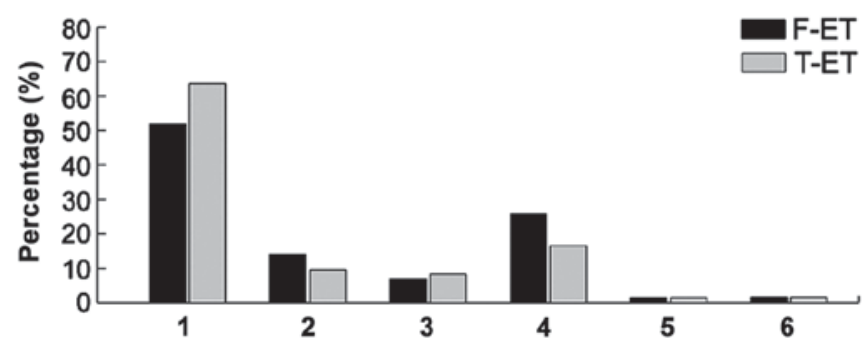

Figure 2. Causes of infertility and embyo transfer cycles (1, tubal problems; 2 , endometriosis; 3 , ovulation disorders; 4, male sperm abnormalities; 5 , other factors; 6 , unknown causes).

the IVF-ET and ICSI-ET group, respectively, while 307 cases $(66.88 \%)$ and 152 cases $(33.12 \%)$ were recorded in the F-ET and T-ET group, respectively. For twin births, 116 cases $(58.29 \%)$ and 83 cases $(41.71 \%)$ were recorded in the IVF-ET and ICSI-ET group, respectively, and 139 cases (69.85\%) and 60 cases $(30.15 \%)$ were recorded, respectively. There were no statistically significant differences between single births or twin births in the corresponding groups $(\mathrm{P}>0.05)$ (Table II).

Child-bearing age of patients in different groups. Patients' child-bearing ages in different groups were divided into 5 age ranges with five-year intervals. The number of patients in the 30-34 years old range was the largest, with $44.03 \%$ (166 cases) in the IVF-ET group, $46.26 \%$ (130 cases) in the ICSI-ET group, $45.07 \%$ (201 cases) in the F-ET group, and $45.28 \%$ (96 cases) in the T-ET group. The differences between groups were not statistically significant $(\mathrm{P}>0.05)$. There were no significant differences between groups in the other age ranges $(\mathrm{P}>0.05)$ (Table III).

Causes of infertility for patients in different groups. Among 658 patients with infertility, 366 cases were infertile due to tubal problems (congenital anomalies of the fallopian tubes, tubal inflammation, and history of tubal ligation), 83 cases due to endometriosis, 44 cases due to ovulation disorders, 150 cases due to male sperm abnormalities (oligoasthenoterazoospermia, necrospermia, and azoospermia), 8 cases due to other factors (abnormal chromosome, and congenital malformation of genital tract), and 7 cases due to unknown causes. Tubal problems ranked the first cause of infertility in all groups, accounting for $66.7 \%$ in the IVF-ET group, $41.5 \%$ in the ICSI-ET group, $52.1 \%$ in the F-ET group, and $63.7 \%$ 
Table IV. Correlation of different fertilization modes with hypertensive disorders in pregnancy (n, \%).

\begin{tabular}{|c|c|c|c|c|c|c|}
\hline \multirow[b]{2}{*}{ Group } & \multicolumn{3}{|c|}{ Gestational hypertension } & \multicolumn{3}{|c|}{ Pre-eclampsia } \\
\hline & All & Single birth & Twin births & All & Single birth & Twin births \\
\hline IVF-ET & $8(2.12)$ & $6(2.30)$ & $2(1.72)$ & $18(4.77)$ & $11(4.21)$ & $7(6.03)$ \\
\hline ICSI-ET & $13(4.63)$ & $5(2.53)$ & $8(9.64)$ & $16(5.69)$ & $8(4.04)$ & $8(9.64)$ \\
\hline OR & $\begin{array}{c}2.01 \\
0.81-4.74\end{array}$ & $\begin{array}{c}1.23 \\
0.35-3.39\end{array}$ & $\begin{array}{c}6.24 \\
1.02-27.5\end{array}$ & $\begin{array}{c}1.36 \\
0.42-4.18\end{array}$ & $\begin{array}{c}0.87 \\
0.25-2.06\end{array}$ & $\begin{array}{c}2.21 \\
0.59-5.26\end{array}$ \\
\hline Corrected $\mathrm{OR}^{\mathrm{a}}$ & $\begin{array}{c}1.69 \\
0.70-4.02\end{array}$ & $\begin{array}{c}0.96 \\
0.29-3.01\end{array}$ & $\begin{array}{c}5.46 \\
0.82-23.6\end{array}$ & $\begin{array}{c}1.17 \\
0.36-3.62\end{array}$ & $\begin{array}{c}0.8 \\
0.26-2.00\end{array}$ & $\begin{array}{c}2.15 \\
0.52-4.78\end{array}$ \\
\hline
\end{tabular}

${ }^{\mathrm{a} C}$ Corrected with patient age, infertility years and BMI.

Table V. Correlation of different embryo transfer cycles with hypertensive disorders in pregnancy (n, \%).

\begin{tabular}{lccccccc}
\hline & \multicolumn{3}{c}{ Gestational hypertension } & & \multicolumn{3}{c}{ Pre-eclampsia } \\
\cline { 2 - 3 } Group & All & Single birth & Twin births & & All & Single birth & Twin births \\
\hline F-ET & $17(3.81)$ & $9(2.93)$ & $8(5.76)$ & & $23(5.16)$ & $15(4.89)$ & $8(5.76)$ \\
T-ET & $4(1.89)$ & $2(1.32)$ & $2(3.33)$ & & $11(5.19)$ & $4(2.63)$ & $7(11.67)$ \\
OR & 0.44 & 0.33 & 0.68 & & 0.93 & 0.52 & 1.86 \\
& $0.13-1.34$ & $0.05-1.47$ & $0.15-3.32$ & & $0.42-1.96$ & $0.20-1.61$ & $0.64-5.61$ \\
Corrected OR & 0.49 & 0.41 & 0.6 & & 0.98 & 0.61 & 1.98 \\
& $0.15-1.51$ & $0.09-1.98$ & $0.13-3.15$ & & $0.44-2.12$ & $0.22-1.84$ & $0.73-6.43$ \\
\hline
\end{tabular}

in the T-ET group. Male sperm abnormalities accounted for $39.86 \%$ (112 cases) in the ICSI-ET group, $10.08 \%$ (38 cases) in the IVF-ET group, $25.78 \%$ (115 cases) in the F-ET group, and $16.51 \%$ (35 cases) in the T-ET group. Apparently, the majority of patients in the ICSI-ET group suffered from male sperm abnormalities (Figs. 1 and 2).

Correlation of different fertilization modes with hypertensive disorders in pregnancy. Among all the 658 maternity patients who conceived through IVF, there were 56 cases $(8.51 \%)$ of hypertensive disorders in pregnancy, including 21 cases $(3.19 \%)$ of gestational hypertension, 34 cases $(5.17 \%)$ of pre-eclampsia and 1 case $(0.15 \%)$ of eclampsia. Among the 459 single-birth patients, there were 30 cases $(6.54 \%)$ of hypertensive disorders in pregnancy, including 11 cases $(2.40 \%)$ of gestational hypertension, 19 cases $(4.14 \%)$ of pre-eclampsia and no eclampsia. Among the 199 twin-birth patients, there were 26 cases $(13.07 \%)$ of hypertensive disorders in pregnancy, including 10 cases $(5.03 \%)$ of gestational hypertension, 15 cases $(7.54 \%)$ of pre-eclampsia and 1 case $(0.50 \%)$ of eclampsia.

Correlation of different fertilization modes with hypertensive disorders in pregnancy. Among the 377 patients in the IVF-ET group, there were 26 cases $(6.90 \%)$ of hypertensive disorders in pregnancy, including 8 cases $(2.12 \%)$ of gestational hypertension, 18 cases $(4.77 \%)$ of pre-eclampsia and no eclampsia. Among the 281 patients in the ICSI-ET group, there were
30 cases $(10.68 \%)$ of hypertensive disorders in pregnancy, including 13 cases $(4.63 \%)$ of gestational hypertension, 16 cases $(5.69 \%)$ of pre-eclampsia and 1 case $(0.36 \%)$ of eclampsia.

The odds ratio (OR) of gestational hypertension in the comparison between the ICSI-ET and the IVF-ET groups was 2.01 (0.81-4.74), and was reduced to 1.69 (0.70-4.02) after correction. There were significant differences in the correlation. The odds ratio of pre-eclampsia in the comparison between the ICSI-ET and IVF-ET groups was 1.36 (0.42-4.18), and was reduced to $1.17(0.36-3.62)$ after correction. There were no significant differences in the correlation. The differences of OR of gestational hypertension or pre-eclampsia in twin-birth patients of the two groups were statistically significant, but the differences in single-birth patients were not statistically significant (Table IV).

Correlation of different embryo transfer cycles with hypertensive disorders in pregnancy. Among the 466 patients in the F-ET group, there were 40 cases $(8.97 \%)$ of hypertensive disorders in pregnancy, including 17 cases (3.81\%) of gestational hypertension, 23 cases $(5.16 \%)$ of pre-eclampsia and no eclampsia. Among the 212 patients in the T-ET group, there were 15 cases $(7.08 \%)$ of hypertensive disorders in pregnancy, including 4 cases $(1.89 \%)$ of gestational hypertension, 11 cases $(5.19 \%)$ of pre-eclampsia and 1 case $(0.47 \%)$ of eclampsia.

The odds ratio (OR) of gestational hypertension in the comparison between the F-ET and T-ET groups was 0.44 
(0.13-1.34), and became 0.49 (0.15-1.51) after correction. The odds ratio of pre-eclampsia in the comparison between the F-ET and T-ET groups was 0.93 (0.42-1.96), and became 0.98 (0.44-2.12) after correction. There were no significant differences in the correlation. The differences of OR of gestational hypertension or pre-eclampsia in single-birth or twin-birth patients of the two groups were not statistically significant (Table V).

\section{Discussion}

In the past 30 years, with commercialization of assisted reproductive technology and vigorous development of its derived technologies, the assisted reproductive technology has advanced from IVF-ET to ICSI-ET, preimplantation genetic diagnosis (PGD), and frozen-thawed embryo transfer. Nevertheless, the test-tube baby technology was considered to be a double-edged sword; on one hand, it was great news for patients suffering from infertility, but at the same time it was plagued with its potential safety risks. Results obtained from prior studies showed that the increase in the number of testtube babies has led to an upsurge in the number of babies with birth defects (4-6). In addition, maternity health at different stages of pregnancy has been adversely affected (7).

Patient characteristics in successful cases of IVF technology. In terms of age, the average maternal age for patients participated in this study was higher than the average in China. In our society, more and more women start pregnancy and give birth at advanced maternal age. Advanced maternal age might be a leading factor for poor outcomes for both mother and newborns. In this study, we discovered that in most cases we had a single birth (69.76\%), and patients who successfully gave twin births were mostly young with good ovarian response, and who provided more eggs and thus more transferable embryos. In terms of mode of delivery, the rate of spontaneous labor was $5.32 \%$ among those patients who conceived successfully through IVF in our hospital's assisted reproductive center. This rate was apparently lower than that of natural pregnancy in China. In this study, we also found that the rate of caesarean section among patients who conceived through IVF was significantly higher than that among patients who conceive naturally. This finding was similar to results reported in prior studies $(8,9)$. This phenomenon can be explained by the fact that in most cases, older patients are those who usually opt for assisted reproductive technology. In view of many potential complications due to advanced maternal age, medical practitioners may relax the cesarean section indications appropriately in their real work. Moreover, those patients who feel a desperate need to have a baby may proactively ask for a caesarean birth.

Causes of infertility for patients who conceived through $I V F$. The number of patients suffering from infertility is increasing. It has been shown that the first three causes of infertility for patients who conceived through IVF are tubal problems, male sperm abnormalities and endometriosis. The tubal problems include congenital tubal abnormalities, tubal non-specific inflammation, and history of tubal ligation of the fallopian tube. Male sperm abnormalities include azoospermia, necrospermia, teratozoospermia, asthenospermia, and oligospermia. This was followed by endometriosis, ovulation disorder, and other causes of infertility (including chromosome abnormalities, male ejaculation disorder, and congenital malformation of genital tract) (10). In this study, we had only few cases $(5.83 \%)$ with ovulation disorder conceived through fresh embryo transfer. The reason can be explained by the fact that patients with ovulation disorder were often with polycystic ovary syndrome (PCOS) and susceptible to ovarian hyperstimulation syndrome (OHSS). For these patients, frozen-thawed embryo transfer could achieve higher live birth rate in comparison with fresh embryo transfer. Additionally, risk of OHSS and incidence of unexpected abortion were also low (11).

Correlation of procedures of in vitro fertilization with hypertensive disorders in pregnancy. Different from natural pregnancy, maternity patients who conceived through IVF may experience adverse pregnancy outcomes such as hypertensive disorders in pregnancy due to biological, medical and sociological factors. Hypertensive disorders in pregnancy usually occur during pregnancy with unknown pathogenesis, and in recent years, the incidence rate of these disorders is on the rise (12), and is one of the top five causes leading to maternal death. According to an incomplete statistic, approximately 60,000 pregnant women die every year because of preeclampsia and eclampsia (13). Currently it is widely accepted that hypertensive disorders in pregnancy may be related to inflammatory factors, genetic factors, placental dysfunction, immune system dysfunction and other mechanisms (14). According to published literature, risk factors include family history, advanced maternal age, history of hypertension, first birth, metabolic syndrome, and twin pregnancy (15). Whether or not the assisted reproductive technology increases the incidence of hypertensive disorders in pregnancy remained contentious in literature.

Mukhopadhaya and Arulkumaran (16) and Sibai (17) believe that the IVF technology increase the risk of adverse pregnancy outcomes such as maternal pre-eclampsia and gestational hypertension. While Sun et al (18) as well as Isaksson et al (19) offer an opposite opinion. In this study, we discovered that incidence of hypertensive disorders in pregnancy was $8.51 \%$, which indicated the IVF technology might affect occurrence and progression of hypertensive disorders in pregnancy at different levels. Other studies showed that age was one of the most important risk factors. Maternity patients at advanced maternal age experienced a high risk of developing hypertensive disorders in pregnancy in comparison with patients at normal maternal age (20). Patients who conceived through IVF usually had an infertility history, and were extremely nervous. This nervousness may lead to anxiety or depression during pregnancy. The stress experienced by patients may increase resistance of the uterine artery, which is one of the factors leading to pre-eclampsia (21).

The procedure of superovulation as part of IVF technology was not able to fully simulate the physiological state of hormone. It was reported that onset of pre-eclampsia may be related to the unusually high levels of HCG on the trigger day which activated the renin-angiotensin-aldosterone system (22). HCG-induced high hormone levels post the trigger day also 
played a role in occurrence of pre-eclampsia. Moreover, one of the most common complications related to the IVF technology is the multiple pregnancy. A multiple pregnancy often increase uterine pressure to an unusually high level, which cause severe placental ischemia and hypoxia, resulting in vascular endothelial cell damage and dysfunction. Thus, a multiple pregnancy is a risk factor for hypertensive disorders in pregnancy (23). It is also arguable that the embryos obtained from donated eggs were allogeneic to the pregnant mother, thus immune system attacks inflict damage to the body, and increase the risk of hypertensive disorders in pregnancy (24).

In this study, the incidence of hypertensive disorders in pregnancy was higher in the ICSI-ET group than that in the IVF-ET group. The difference was statistically significant, and the difference was even more significant in the comparison of twins in the two groups. This indicates that the ICSI-ET technology, to a certain extent, increases the risk of hypertensive disorders in pregnancy. Unlike the routine IVF-ET technology, following the ICSI-ET protocol, a single normal sperm was placed in a fine needle under a microscope using external force, followed by piercing of the zona pellucida and the egg membrane with the needle tip and injection of the sperm into the follicle pulp to complete fertilization. In this process of fertilization, the invasive procedure can create some unfavorable conditions that may impact the implantation of the embryos and change the endometrial microenvironment, which can affect the health of the offspring (25).

In this study, we discovered that frozen-thawed embryo transfer did not increase the risk of developing hypertensive disorders in pregnancy. Unlike the fresh embryo transfer technology, following the frozen-thawed embryo transfer protocol, the early embryos were frozen for storage, and were resuscitated before transfer. This technique can increase the cumulative pregnancy rate of a single ovulation induction/egg retrieval process. It also allows recovery of the ovary and reduces patients suffering from multiple periods of egg retrieval, and therefore it is convenient and economical to patients.

We concluded that the risk of onset of hypertensive disorders in pregnancy has a certain correlation with the ICSI fertilization technology, however no apparent correlation with transfer cycles was detected.

\section{Acknowledgements}

This study was supported by the National Natural Science Fund 8160060698 .

\section{References}

1. Myers ER, McCrory DC, Mills AA, Price TM, Swamy GK Tantibhedhyangkul J, Wu JM and Matchar DB: Effectiveness of assisted reproductive technology (ART). Evid Rep Technol Assess (Full Rep) 167: 1-195, 2008.

2. Pinborg A: IVF/ICSI twin pregnancies: Risks and prevention. Hum Reprod Update 11: 575-593, 2005.

3. Ahmad AS and Samuelsen SO: Hypertensive disorders in pregnancy and fetal death at different gestational lengths: A population study of 2121371 pregnancies. BJOG 119: 1521-1528, 2012.
4. Paoloni-Giacobino A: Epigenetics in reproductive medicine. Pediatr Res 61: R51-R57, 2007.

5. Kanyó K and Konc J: A follow-up study of children born after diode laser assisted hatching. Eur J Obstet Gynecol Reprod Biol 110: 176-180, 2003.

6. Halliday J, Oke K, Breheny S, Algar E and J Amor D: BeckwithWiedemann syndrome and IVF: A case-control study. Am J Hum Genet 75: 526-528, 2004.

7. Wang YA, Chughtai AA, Farquhar CM, Pollock W, Lui K and Sullivan EA: Increased incidence of gestational hypertension and preeclampsia after assisted reproductive technology treatment. Fertil Steril 105: 920-926.e2, 2016.

8. Hammarberg K, Fisher JR and Rowe HJ: Women's experiences of childbirth and post-natal healthcare after assisted conception. Hum Reprod 23: 1567-1573, 2008.

9. Baxi A and Kaushal M: Outcome of twin pregnancies conceived after assisted reproductive techniques. J Hum Reprod Sci 1: 25-28, 2008.

10. Kintiraki E, Papakatsika S, Kotronis G, Goulis DG and Kotsis V: Pregnancy-induced hypertension. Hormones (Athens) 14: 211-223, 2015.

11. Chen ZJ, Shi Y, Sun Y, Zhang B, Liang X, Cao Y, Yang J, Liu J, Wei $\mathrm{D}$, Weng $\mathrm{N}$, et al: Fresh versus frozen embryos for infertility in the polycystic ovary syndrome. N Engl J Med 375: 523-533, 2016.

12. Kuklina EV, Ayala C and Callaghan WM: Hypertensive disorders and severe obstetric morbidity in the United States. Obstet Gynecol 113: 1299-1306, 2009.

13. Jido TA and Yakasai IA: Preeclampsia: A review of the evidence. Ann Afr Med 12: 75-85, 2013.

14. Stern JE, Luke B, Tobias M, Gopal D, Hornstein MD and Diop H: Adverse pregnancy and birth outcomes associated with underlying diagnosis with and without assisted reproductive technology treatment. Fertil Steril 103: 1438-1445, 2015.

15. Owiredu W, Ahenkorah L, Turpin CA, Amidu N and Laing EF: Putative risk factors of pregnancy-induced hypertension among Ghanaian pregnant women. J Med Biomed Sci 1: 62-76, 2012.

16. Mukhopadhaya N and Arulkumaran S: Reproductive outcomes after in-vitro fertilization. Curr Opin Obstet Gynecol 19: 113-119, 2007.

17. Sibai BM: Subfertility/infertility and assisted reproductive conception are independent risk factors for pre-eclampsia. BJOG 122: 923, 2015.

18. Sun LM, Walker MC, Cao HL, Yang Q, Duan T and Kingdom JC: Assisted reproductive technology and placenta-mediated adverse pregnancy outcomes. Obstet Gynecol 114: 818-824, 2009.

19. Isaksson R, Gissler M and Tiitinen A: Obstetric outcome among women with unexplained infertility after IVF: A matched casecontrol study. Hum Reprod 17: 1755-1761, 2002.

20. Tabcharoen C, Pinjaroen S, Suwanrath C and Krisanapan O: Pregnancy outcome after age 40 and risk of low birth weight. J Obstet Gynaecol 29: 378-383, 2009.

21. Kurki T, Hiilesmaa V, Raitasalo R, Mattila $\mathrm{H}$ and Ylikorkala $\mathrm{O}$ Depression and anxiety in early pregnancy and risk for preeclampsia. Obstet Gynecol 95: 487-490, 2000.

22. Mikat B, Zeller A, Scherag A, Drommelschmidt K, Kimmig R and Schmidt M: $\beta$ hCG and PAPP-A in first trimester: Predictive factors for preeclampsia? Hypertens Pregnancy 31: 261-267, 2012.

23. Miyake H, Iwasaki N, Nakai A, Suzuki S and Takeshita T: The influence of assisted reproductive technology on women with pregnancy-induced hypertension: A retrospective study at a Japanese Regional Perinatal Center. J Nippon Med Sch 77: 312-317, 2010.

24. Pecks U, Maass N and Neulen J: Oocyte donation: a risk factor for pregnancy-induced hypertension: a meta-analysis and case series. Dtsch Arztebl Int 108: 23-31, 2011.

25. Buzzi PJ, Zappacosta MP, Auge L, Isa L, Obejero EY and Bello A: Beneficial effect of local injury to the endometrium in intracytoplasmic sperm injection (ICSI) patients with recurrent implantation failure. Fertil Steril 102: e293, 2014. 